IRSTI 31.21 .29

\author{
A.B. Niyazbekova, T.A. Shakirov \\ Zhangir khan West Kazakhstan agrarian-technology University, Uralsk, Kazakhstan \\ e-mail: abnyazbekova@mail.ru, shakirov_1985@mail.ru
}

\title{
Study of the effectiveness of mixed organo-inorganic inhibitors
}

\begin{abstract}
The relevance of the study lies in the fact that industrial production requires inhibitors with a high degree of protection, low cost of production and meet the requirements for chemical reagents. Individual organic or inorganic inhibitors used in production do not always meet the above requirements. The mutual influence of mixtures of various inhibitors was considered by V.P. Barannik. Using the concepts developed in the theory of catalysis for mixed catalysts, he established that the action of inhibitor mixtures in many respects resembles the action of catalyst mixtures. For mixtures of inhibitors, the effects of mutual amplification are established - synergism of the protective action, additivity, and the effect of mutual weakening - antagonism of the protective action. Strengthening the protective action of an organic corrosion inhibitor is possible by creating a composition of this inhibitor with other substances that enhance its action or by introducing into the molecule of this organic substance functional groups with heteroatoms different in charge (for example, N, O, P). The study and creation of mixed organoinorganic compositions of inhibitors, mutually reinforcing the action of each other and capable of forming self-organizing surface layers is a promising and little-studied area. For research as main components of the inhibiting compositions taken: sodium dihydrogen phosphate, sodium hydrogen phosphate, sodium orthophosphate, sodium dihydrogendiphosphate, diphosphate sodium, sodium cyclotriphosphate and sodium cyclohexaphosphate, urea, thiourea, diethylamine hydrochloric acid, monoethanolamine, triethanolamine. The research was conducted according to the methods of state standards. The research methods were gravimetry, photocolorimetry, potentiometry. Experimentally determined quantitative indicators of the corrosion process: the rate of the corrosion process, degree of protection, depth indicator, braking coefficient. Analysis of the experimental data allows us to establish the laws of change in the quantitative indicators of the corrosion process and the stability of the resulting protective film on the metal surface. The experimental data obtained correlate well with the calculated data of the thermodynamic parameters of the corrosion process. Based on the research conducted, the chemical process and the mechanism of the interaction of the inhibitor with the surface of the metal plate were established. The evaluation of the stability of the resulting film was carried out on a scale of corrosion resistance in relation to steel St-3. On the basis of the obtained data, it was established that the anticorrosion efficiency depends on the nature of the organic and inorganic component and the ratios of the components of the systems. Mathematical and static data processing was carried out by Student's method with a confidence interval of 0.95 . The regularities established during the work expand and complement the existing ideas about the protective effect of two-component organic-inorganic systems. The results will significantly contribute to the creation of effective, environmentally friendly and cost-effective inhibitors of steel corrosion.
\end{abstract}

Key words: corrosion, inhibitor, phosphate, degree of protection, depth indicator.

\section{Introduction}

In the largest countries of the world, the production of metal corrosion inhibitors is represented by a wide range of chemical products; however, for the industrial production of a certain product, the cost of production and its compliance with the requirements for chemicals are an important factor $[1,2,3]$.

At present, the modification of the surface of metals with inhibitors, containing in its composition compounds capable of forming self-organizing surface layers, is promising and is a little studied area. 
Studying the effectiveness of such systems, consisting of mixed corrosion inhibitors and determining the nature of their interaction is important.

The works of A.Gabitov shown [4] that it is possible to create high-performance environmentally friendly corrosion inhibitors of carbon steels are the most studied compounds, aromatic and aliphatic amines. Unfortunately, the reagents used do not always provide a sufficiently high protective effect.

For most organic inhibitors characterized by an increase in their concentration to a certain amount of increase in the effectiveness of the protective action, and then the effectiveness does not change. In inorganic anode inhibitors at low concentrations there is an increase in the corrosion rate and only when a certain concentration is reached there is a sharp decrease in the corrosion rate due to the passivation of the metal surface.

Of the inorganic inhibitors, phosphoric acid and its salts are the most promising for corrosion suppression, but their inhibitory properties are manifested only at fairly high concentrations.

A promising direction of creation of new effective metal corrosion inhibitors for aqueous media is the use of sodium phosphates, which in condensed form are in the waste of phosphorus production - the liquid phase of poor phosphorus sludge, the so-called "cottrel milk".

The inhibitory effect of sodium polyphosphate may be due to the ability of polyphosphates to prevent the reduction of oxygen on the surface of iron, thereby facilitating the adsorption of dissolved oxygen, which leads to the passivation of the metal.

Currently, there are two ways to obtain inhibitors: the first way - the creation of individual inhibitory compounds by chemical synthesis using the knowledge of the mechanism of action of inhibitors and the relationship of protective action with the structure of molecules; the second way - to obtain combined inhibitors, especially on the basis of waste chemical and petrochemical industries.

The first way is characterized by high cost and complexity of obtaining individual compounds. These disadvantages are deprived of the second way, however, in this case, for the purposeful creation of combined inhibitors, it is necessary to know the patterns of mutual influence of their components with a joint action on the corrosion process.

Even in the conditions of one field at different sites, this figure may vary significantly. This may be due to the solubility (dispersibility) of the inhibitor in formation fluids, its low degree of compatibility with formation waters, incorrect selection of the reagent for specific conditions. Usually in practice, this problem is solved by increasing the dosage of the reagent, which also does not always give the desired effect.

Therefore, it is necessary to create new inhibitor compositions that could provide a high protective effect in a wide range of conditions of use or improve the quality of existing formulations.

The mutual influence of mixtures of different inhibitors was first studied in detail by V.Barannik [5]. Using the concepts developed in the theory of catalysis for mixed catalysts, he found that the action of inhibitor mixtures is largely similar to the action of catalyst mixtures [6]. For mixtures of inhibitors, the effects of mutual reinforcement were established - synergism of the protective action, additivity and the effect of mutual weakening - antagonism of the protective action.

Strengthening the protective action of an organic corrosion inhibitor can, in principle, be achieved in two ways: creating a composition of this inhibitor with other substances that enhance its action and introducing into the molecule of this organic substance functional groups with heteroatoms differing in charge (for example, N, O, P). In the first case, they usually speak of intermolecular synergism, in the second case, intramolecular $[7,8]$.

The essence of this effect is that one or two functional groups with a heteroatom are introduced into the inhibitor molecule, which differ in size and sign of charge from the one available in the molecule. In this case, one group can behave like a cation, the other - like anion. Examples of such inhibitors may be substances containing $-\mathrm{NH}_{2}$ and $-\mathrm{OH},-\mathrm{NH}_{2}$ and $-\mathrm{SH}$, $-\mathrm{NH}_{2}$ and $-\mathrm{COOH}$ groups. When molecules of such a substance are adsorbed on a metal, some of them are adsorbed by one group (for example, by an amino group), and some by another (for example, by a hydroxy group). This leads to a decrease in repulsive forces between molecules on the metal surface and contributes to the formation of a more dense inhibitor film.

\section{Materials and methods}

To assess the effectiveness of the inhibitory effect of mixed organo-inorganic compositions, the study was conducted at a concentration of $0.01 \mathrm{~mol} / \mathrm{dm}^{3}$ at a ratio of reagents in the mixture: 1:3, 1:1 and 3:1. As the main elements inhibiting compositions taken: sodium dihydrogen phosphate, sodium hydrogen phosphate, sodium orthophosphate, sodium dihydrogendiphosphate, diphosphate sodium, sodium cyclotriphosphate and sodium cyclohexaphosphate, urea, 
thiourea, diethylamine hydrochloric acid, monoethanolamine, triethanolamine. Corrosion tests were carried out according to the methods of state standards on steel St-3 composition $[9,10,11]$. The duration of the experiments ranged from $24-480$ hours. The following research methods were used: gravimetry, photocolorimetry, potentiometry. Studies were carried out in comparison with control samples of individual inorganic and organic inhibitors.

Quantitative indicators of corrosion processes were calculated by the formulas; the evaluation of measurement uncertainty was carried out accord- ing to an algorithm using Student's coefficient [12, 13].

\section{Results and Discussion}

In the study of the sodium dihydrophosphate system in a mixture with organic components (Figure 1), it was found that in two-component $\mathrm{NaH}_{2} \mathrm{PO}_{4}$ systems with urea, thiourea, diethylamine, with a $3: 1$ ratio of components, changes in the corrosion rate are insignificant. The system $\mathrm{NaH}_{2} \mathrm{PO}_{4}$-urea shows increased protective effect $(Z=79.92 \%)$.

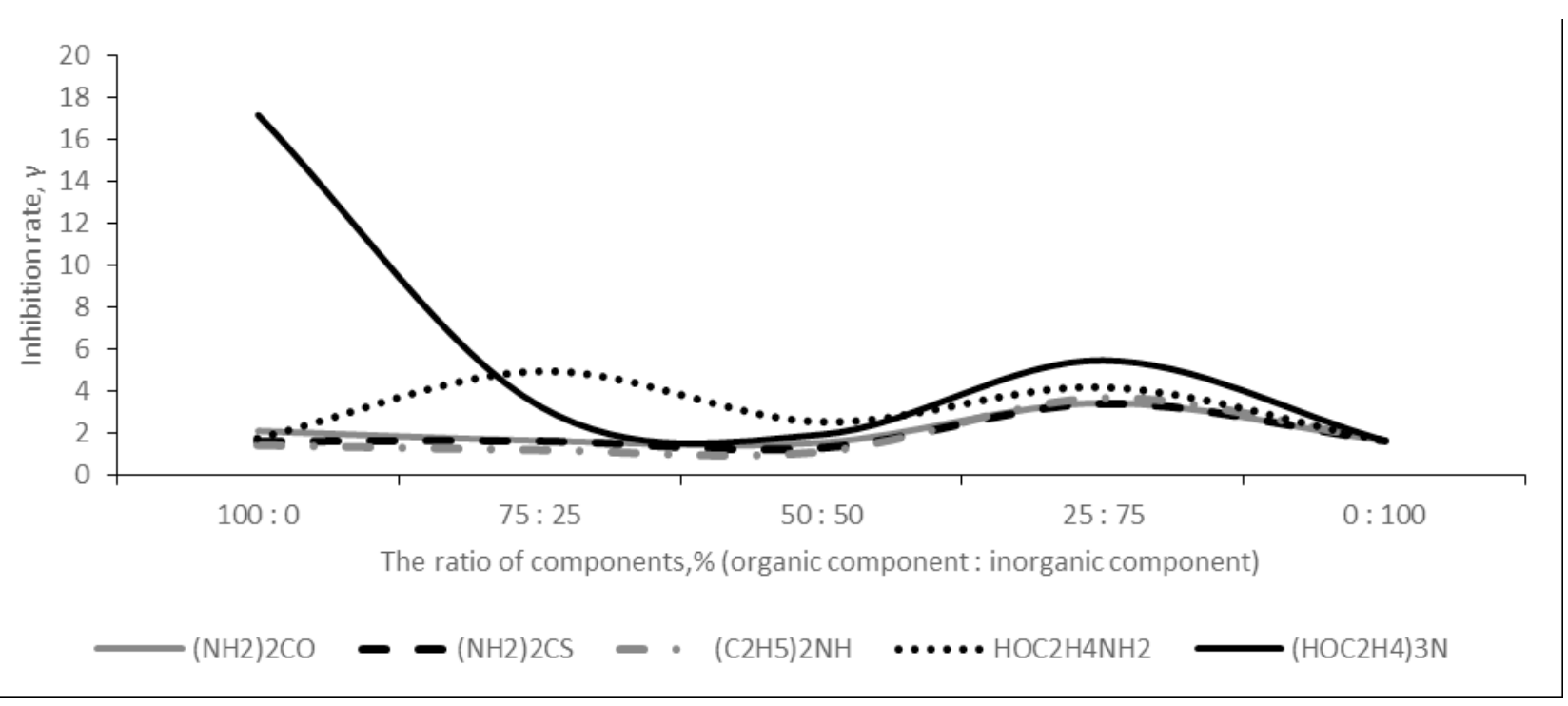

Figure 1 - Inhibition of St-3 steel corrosion in systems with sodium dihydrophosphate $\mathrm{NaH}_{2} \mathrm{PO}_{4}\left(\mathrm{C}_{\mathrm{m}}=0.01 \mathrm{~mol} / \mathrm{dm}^{3}\right)$

Observations have shown that in systems with a ratio of 1:1 component in the presence of organic additives, the protective effect is similar in all systems. For systems with a 1:3 ratios, an increase in the protective effect of 3.5 times was observed, in contrast to a one-component system containing only an inorganic inhibitor.

Studies have shown that in stationary conditions, the addition of an organic component to $\mathrm{NaH}_{2} \mathrm{PO}_{4}$ leads to a decrease in the corrosion rate, steel compared to the control sample by 2-3 times.

In the $\mathrm{Na}_{2} \mathrm{HPO}_{4}$ systems with organic components (Figure 2), according to the results of gravimetric tests, it was revealed that in clean environments there is a high rate of the corrosion process and, accordingly, a low inhibition coefficient. Comparison of changes in the rates of corrosion processes of a system with a pure inorganic inhibitor and systems with a ratio of $3: 1$ components showed similar values of the corrosion rate constants and inhibition coefficients. In the system $\mathrm{Na}_{2} \mathrm{HPO}_{4}$ - triethanolamine, the protective effect was reduced by a factor of 1.5 from 94.61 to 65.08 , and in systems with a 1:1 ratio of components, the protective effect did not change compared to a pure phosphate inhibitor. In the $\mathrm{Na}_{2} \mathrm{H}-$ $\mathrm{PO}_{4}-\left(\mathrm{HOC}_{2} \mathrm{H}_{4}\right)_{3} \mathrm{~N}$ system with a 1:3 ratio of components, a sharp increase in the protective effect is observed, as evidenced by comparing the values of inhibition coefficients in this system (79.33) and in the system containing pure $\mathrm{Na}_{2} \mathrm{HPO}_{4}$ (4.67).

The increase of the protective effect in the system of triethanolamine, apparently due to participation of the lone pairs on the nitrogen atom in the molecule of triethanolamine, that binds the hydrogen atom in the hydrogen phosphate in the donor-acceptor mechanism with the formation of neutral salts. And unlike pure triethanolamine, it is more easily sorbed on the formed phosphate film covering the surface of the 
metal, than increases the protective effect of the entire system:

$$
\mathrm{HPO}_{4}^{2-}+: \mathrm{N}\left(\mathrm{C}_{2} \mathrm{H}_{4} \mathrm{OH}\right)_{3} \rightarrow\left[\mathrm{H}: \mathrm{N}\left(\mathrm{C}_{2} \mathrm{H}_{4} \mathrm{OH}\right)_{3}\right]^{+}+\mathrm{PO}_{4}^{3-}
$$

In the system under consideration, the inhibitor $\mathrm{Na}_{3} \mathrm{PO}_{4}$ and its mixtures with organic components experimental data show that in a single-component system containing only $\mathrm{Na}_{3} \mathrm{PO}_{4}$, high inhibitory properties with a degree of protection of $97,46 \%$ are manifested. The data in the $\mathrm{Na}_{3} \mathrm{PO}_{4}$-diethylamine system in a 1:3 ratio shows a 5-fold decrease in the protective effect. In systems with other organic components with the same ratio, an increase in the protective effect up to $98,24 \%$ is observed in the presence of both urea and triethanolamine.

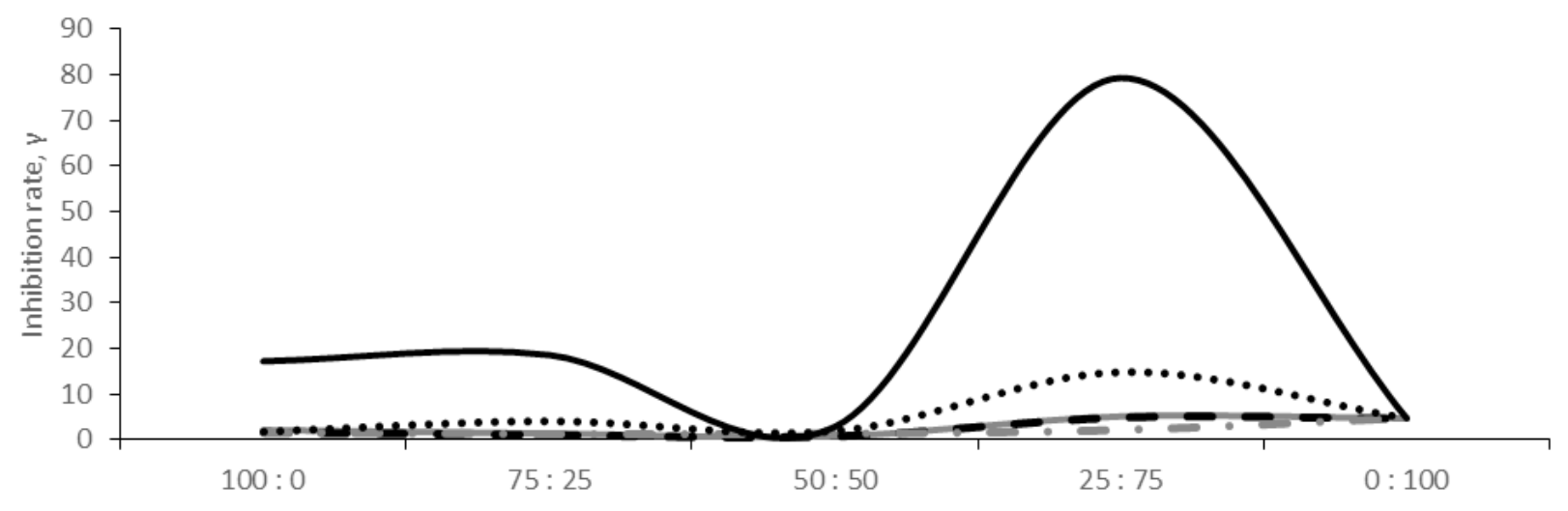

The ratio of components, $\%$ (organic component: inorganic component)

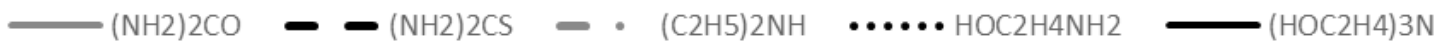

Figure 2 - Inhibition of St-3 steel corrosion in systems with sodium hydroorthophosphate $\mathrm{Na}_{2} \mathrm{HPO}_{4}\left(\mathrm{C}_{\mathrm{m}}=0.01 \mathrm{~mol} / \mathrm{dm}^{3}\right)$

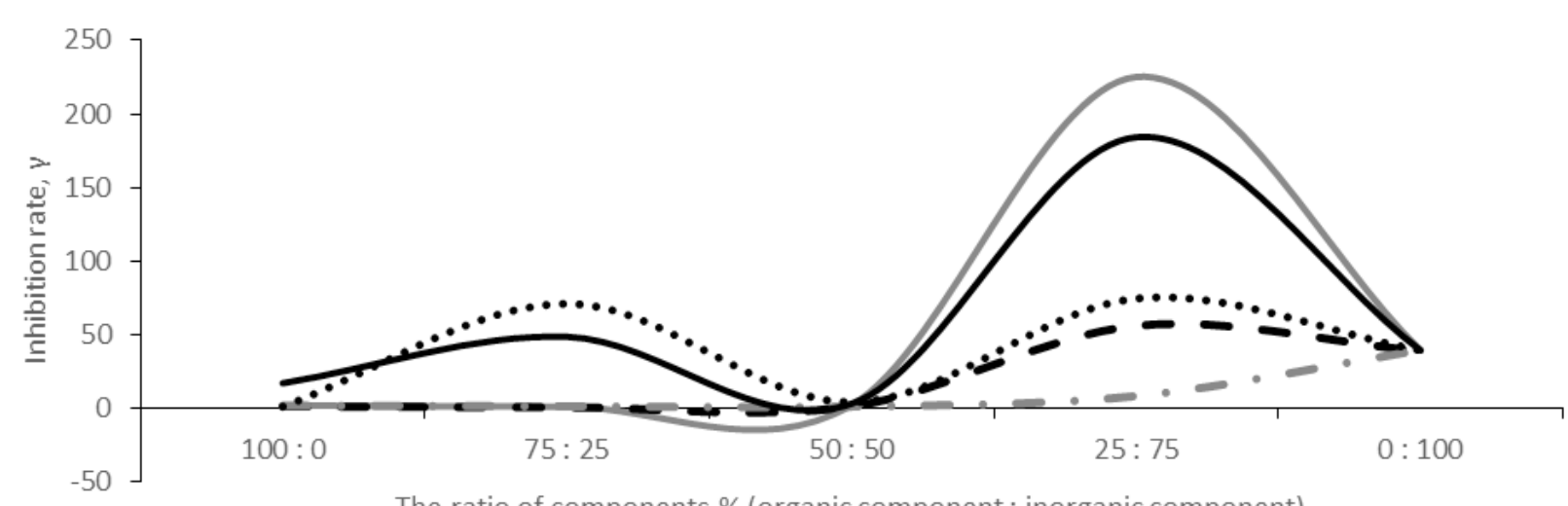

The ratio of components,\% (organic component: inorganic component)

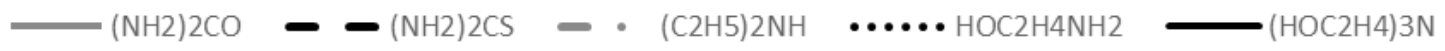

Figure 3 - Inhibition of St-3 steel corrosion in systems with sodium orthophosphate $\mathrm{Na}_{3} \mathrm{PO}_{4}$ $\left(\mathrm{C}_{\mathrm{m}}=0.01 \mathrm{~mol} / \mathrm{dm}^{3}\right)$

With a 1:1 ratio of components, the protective effect is not significant for all systems. In systems with a 3:1 ratio, the protective effect is increased in the following sequence:

Int. j. biol. chem. (Online) urea $>$ triethanolamine $>$ monoethanolamine.

The test results of two-component dihydrodiphosphate inhibitory systems with control samples International Journal of Biology and Chemistry 12, № 1, 181 (2019) 
showed that in all systems of this series, at all ratios of the components, the inhibition coefficient is low and does not exceed 4.

Sodium diphosphate systems with organic substances are also considered according to the main indicators characterizing the course of the corrosion process. Analysis of the data obtained makes it pos- sible to assert that pure diphosphate systems have the least protective ability (Figure 4). The addition of a small amount of the organic component causes a multiple increase in the protective effect of the inhibitor, which can be shown by the example of the diphosphate system with triethanolamine, the protective effect of which is $94.18 \%$.

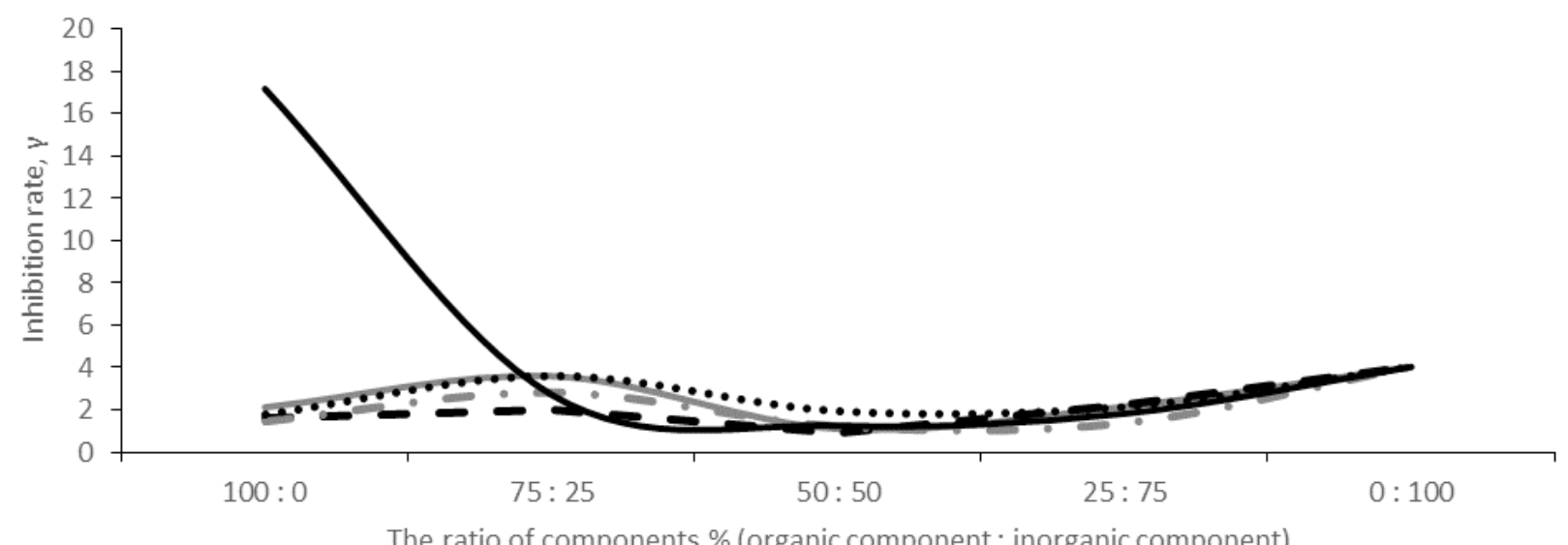

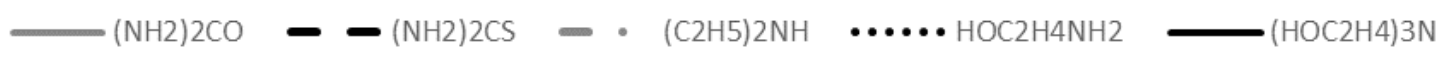

Figure 4 - Inhibition of St-3 steel corrosion in systems with sodium dihydrodiphosphate $\mathrm{Na}_{2} \mathrm{H}_{2} \mathrm{P}_{2} \mathrm{O}_{7}\left(\mathrm{C}_{\mathrm{m}}=0.01 \mathrm{~mol} / \mathrm{dm}^{3}\right)$

The degree of change of the protective effect in two-component systems is influenced by both the nature of organic matter and the ratio of components.
Thiourea was the best supplement in this series. The least strongly protective effect of pure diphosphate is triethanolamine (Figure 5).

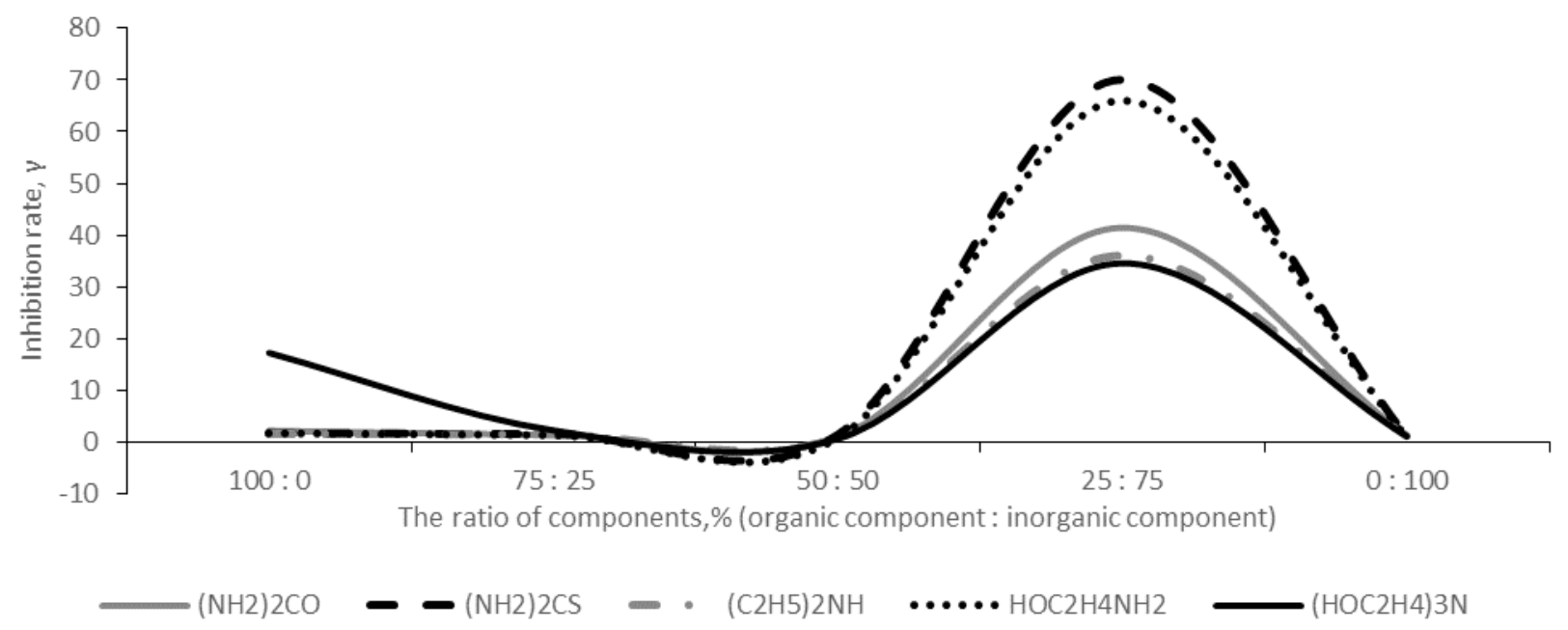

Figure 5 - Inhibition of St-3 steel corrosion in systems with sodium diphosphate $\mathrm{Na}_{4} \mathrm{P}_{2} \mathrm{O}_{7}$ $\left(\mathrm{C}_{\mathrm{m}}=0.01 \mathrm{~mol} / \mathrm{dm}^{3}\right)$ 
Trimetaphosphate systems have also been investigated for inhibitory abilities both in pure form and as mixtures (Figure 6). In all cases, with the exception of pure triethanolamine, the protective effect is relatively low and $\gamma$ does not exceed 5 .

The environment with pure trimetaphosphate compared with the system with triethanolamine 2,2 times lower in inhibiting properties. In systems with trimetaphosphate, the degree of protection is also influenced by the ratio and nature of organic components. The change in the protective properties in a two-component system at different ratios shows an equivalent relationship, but the absolute values differ by an average of 2 times.

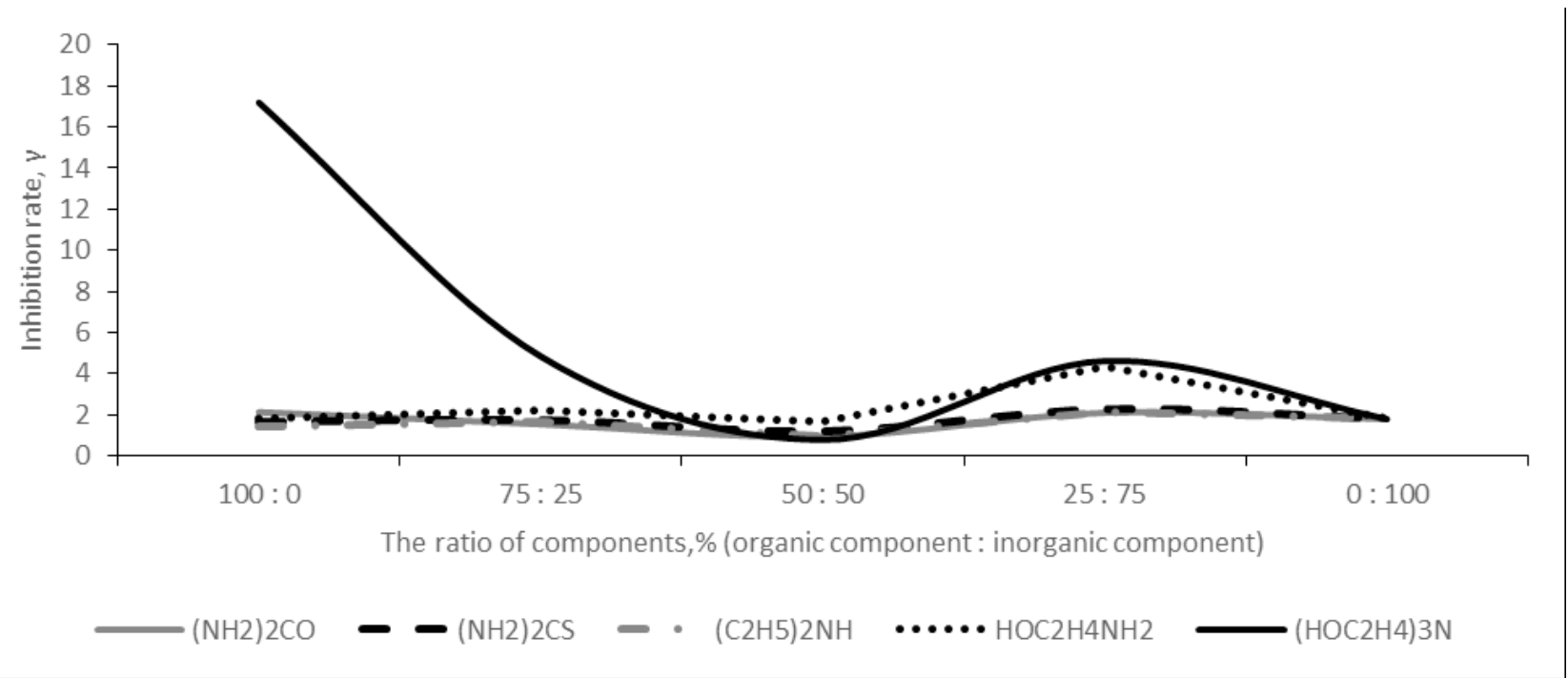

Figure 6 - Inhibition of St-3 steel corrosion in systems with sodium cyclotriphosphate $\mathrm{Na}_{3} \mathrm{P}_{3} \mathrm{O}_{9}\left(\mathrm{C}_{\mathrm{m}}=0.01 \mathrm{~mol} / \mathrm{dm}^{3}\right)$

Pure one-component cyclohexaphosphate system does not exhibit high inhibitory properties (Figure 7). A system containing a purely organic component, triethanolamine, exhibits a protective effect of $94.18 \%$. Two-component hexaphosphate systems showed moderately viable but insignificant absolute values of the protective coefficient (not higher than 4).

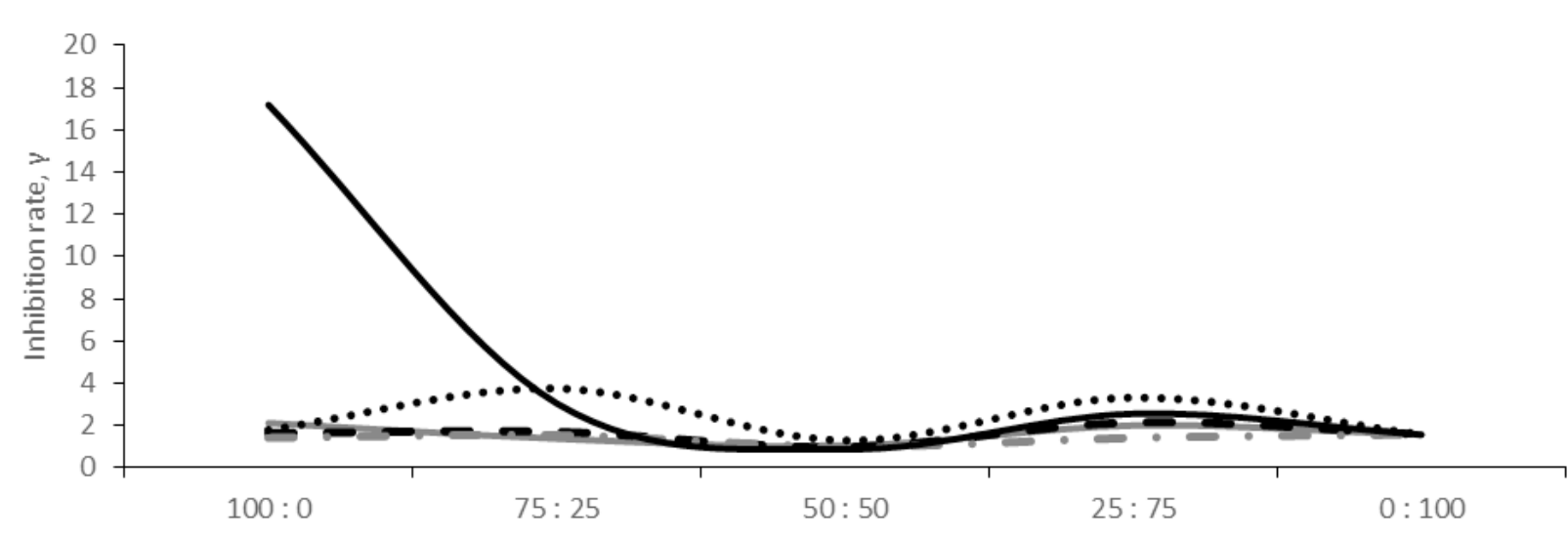

The ratio of components, $\%$ (organic component: inorganic component)

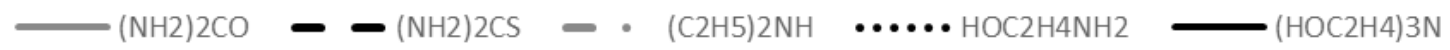

Figure 7 - Inhibition of St-3 steel corrosion in systems with sodium cyclohexaphosphate $\mathrm{Na}_{3} \mathrm{P}_{3} \mathrm{O}_{9}\left(\mathrm{C}_{\mathrm{m}}=0.01 \mathrm{~mol} / \mathrm{dm}^{3}\right)$ 
During the experiment, 118 systems (13 singlecomponent and 105 two-component) were investigated and the dependence of their anti-corrosive effectiveness on the nature of the organic and inorganic components, as well as their ratios. It has been established that, in pure form, sodium orthophosphate and triethanolamine have the most pronounced protective properties, and fully substituted inorganic phosphates with the introduction of a small additive of organic component are most effective.

Of all the systems, antagonism is most pronounced in the sodium hydrophosphate-diethylamine system (Figure 8).

The type of interaction in the considered compositions depends on the ratio of components. A signifi- cant decrease in the protective effect, with respect to pure substances, is observed for all systems with a component ratio of 1:1.

The effectiveness of the protective effect of all considered systems also depends on the $\mathrm{pH}$ of the solution and the total mineralization. With an increase in the $\mathrm{pH}$ of the medium and the overall mineralization, the protective effect increases.

On the basis of the experimental data obtained, it was found that the most practically significant protective effect is 14 two-component (Table 1).

According to the research results, metal resistance was evaluated by a scale of corrosion resistance in relation to the systems under consideration, based on the deep corrosion index $[14,15]$.

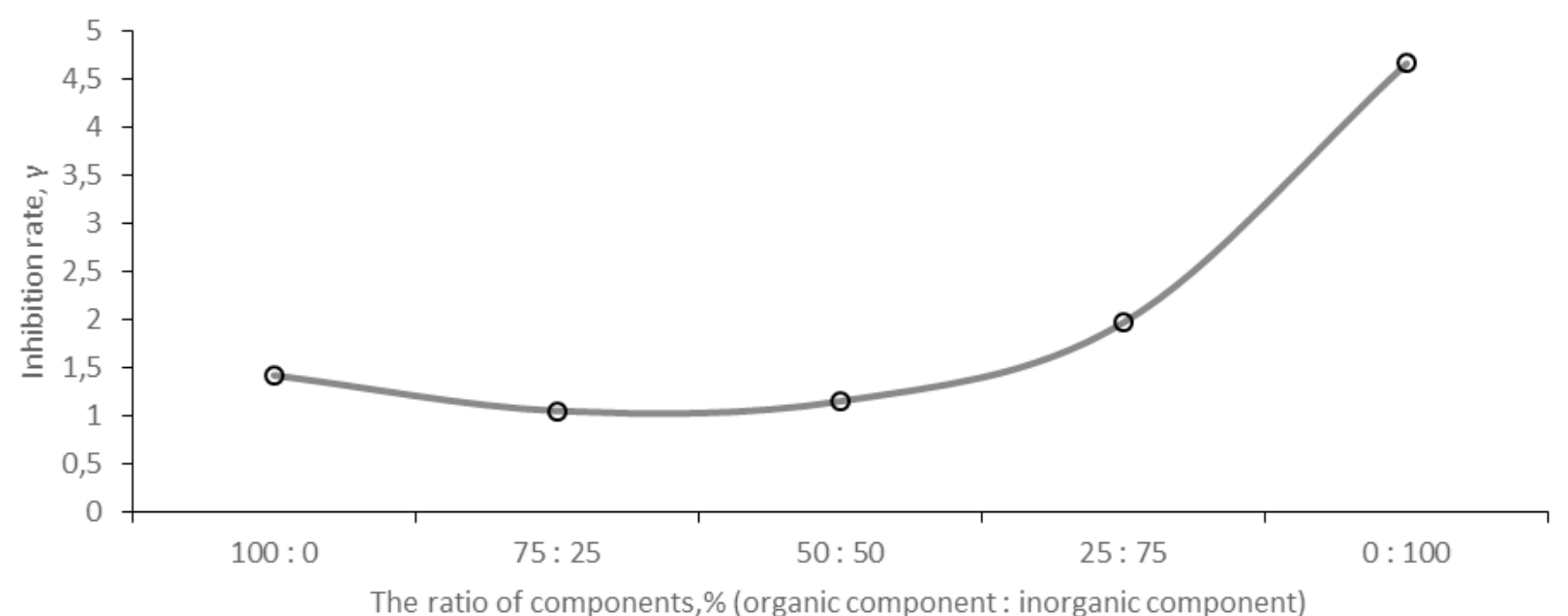

Figure 8 - Antagonism in the system sodium hydrophosphate - diethylamine $\left(\mathrm{C}_{\mathrm{m}}=0.01 \mathrm{~mol} / \mathrm{dm}^{3}\right)$

Table 1 - The most effective systems in terms of corrosion resistance of steel St-3

\begin{tabular}{|c|c|c|c|c|c|}
\hline № & System (ratio) & $\mathrm{pH}$ & $\begin{array}{c}\text { Corrosion rate, } \mathrm{mg} / \\
\mathrm{m}^{2} \cdot \text { hour }\end{array}$ & $\begin{array}{c}\text { Degree of } \\
\text { protection, } \mathrm{Z} \%\end{array}$ & $\begin{array}{l}\text { Inhibitory } \\
\text { effect, } \gamma\end{array}$ \\
\hline \multicolumn{6}{|c|}{ Completely resistant (point - 0) } \\
\hline 1 & $\mathrm{Na}_{3} \mathrm{PO}_{4}+\left(\mathrm{NH}_{2}\right)_{2} \mathrm{CO} \quad(1: 3)$ & 9,09 & $0.51 \pm 0,05$ & 99.56 & 225.17 \\
\hline 2 & $\mathrm{Na}_{3} \mathrm{PO}_{4}+\left(\mathrm{HOC}_{2} \mathrm{H}_{4}\right)_{3} \mathrm{~N} \quad(1: 3)$ & 8,21 & $0.60 \pm 0,02$ & 99.46 & 184.24 \\
\hline \multicolumn{6}{|c|}{ Extremely resistant (point - 1) } \\
\hline 3 & $\mathrm{Na}_{4} \mathrm{P}_{2} \mathrm{O}_{7}+\left(\mathrm{NH}_{2}\right)_{2} \mathrm{CO} \quad(1: 3)$ & 7,13 & $2.9 \pm 0,1$ & 97.59 & 41.43 \\
\hline 4 & $\mathrm{Na}_{3} \mathrm{PO}_{4}+\left(\mathrm{NH}_{2}\right)_{2} \mathrm{CS} \quad(1: 3)$ & 9,39 & $2.1 \pm 0,1$ & 98.24 & 56.67 \\
\hline 5 & $\mathrm{Na}_{4} \mathrm{P}_{2} \mathrm{O}_{7}+\left(\mathrm{NH}_{2}\right)_{2} \mathrm{CS} \quad(1: 3)$ & 9,63 & $1.7 \pm 0,1$ & 98.57 & 70.00 \\
\hline 6 & $\mathrm{Na}_{4} \mathrm{P}_{2} \mathrm{O}_{7}+\left(\mathrm{C}_{2} \mathrm{H}_{5}\right)_{2} \mathrm{NH} \quad(1: 3)$ & 9,53 & $3.3 \pm 0,1$ & 97.23 & 36.04 \\
\hline 7 & $\mathrm{Na}_{3} \mathrm{PO}_{4}+\mathrm{HOC}_{2} \mathrm{H}_{4} \mathrm{NH}_{2} \quad(1: 3)$ & 9,42 & $1.60 \pm 0,06$ & 98.66 & 74.38 \\
\hline
\end{tabular}


Continuation of table 1

\begin{tabular}{|c|cc|c|c|c|c|}
\hline 8 & $\mathrm{Na}_{4} \mathrm{P}_{2} \mathrm{O}_{7}+\mathrm{HOC}_{2} \mathrm{H}_{4} \mathrm{NH}_{2}$ & $(1: 3)$ & 5,53 & $1.8 \pm 0,1$ & 98.49 & 66.11 \\
\hline 9 & $\mathrm{Na}_{2} \mathrm{HPO}_{4}+\left(\mathrm{HOC}_{2} \mathrm{H}_{4}\right)_{3} \mathrm{~N}$ & $(1: 3)$ & 9,01 & $1.5 \pm 0,1$ & 98.74 & 79.33 \\
\hline 10 & $\mathrm{Na}_{4} \mathrm{P}_{2} \mathrm{O}_{7}+\left(\mathrm{HOC}_{2} \mathrm{H}_{4}\right)_{3} \mathrm{~N} \quad(1: 3)$ & 5,41 & $3.4 \pm 0,3$ & 97.1 & 34.54 \\
\hline 11 & $\mathrm{Na}_{3} \mathrm{PO}_{4}+\mathrm{HOC}_{2} \mathrm{H}_{4} \mathrm{NH}_{2}$ & $(3: 1)$ & 10,83 & $1.70 \pm 0,05$ & 98.59 & 70.92 \\
\hline 12 & $\mathrm{Na}_{3} \mathrm{PO}_{4}+\left(\mathrm{HOC}_{2} \mathrm{H}_{4}\right)_{3} \mathrm{~N} \quad(3: 1)$ & 10,83 & $2.4 \pm 0,1$ & 97.95 & 48.71 \\
\hline \multicolumn{7}{|c|}{ Extremely resistant (point - 2) } \\
\hline 13 & $\mathrm{Na}_{2} \mathrm{HPO}_{4}+\mathrm{HOC}_{2} \mathrm{H}_{4} \mathrm{NH}_{2} \quad(1: 3)$ & 3,71 & $8.0 \pm 0,6$ & 93.28 & 14.88 \\
\hline 14 & $\mathrm{Na}_{2} \mathrm{HPO}_{4}+\left(\mathrm{HOC}_{2} \mathrm{H}_{4}\right)_{3} \mathrm{~N}$ & $(3: 1)$ & 9,38 & $6.4 \pm 5,4$ & 94.61 & 18.56 \\
\hline
\end{tabular}

\section{Conclusion}

Thus, of all the studied systems, 14 two-component systems are - very resistant (point 0-2). The relatively low protective effect of two-component systems containing cyclic phosphates is apparently due to the fact that this type of phosphate in a dilute aqueous solution is subject to significant hydrolytic cleavage $[16,17,18]$ with the formation of a large amount of acid salts of ortho- and diphosphoric acids, which leads to increasing the $\mathrm{pH}$ of the solution and a corresponding decrease in the effectiveness of the inhibiting compositions.

On the basis of the experimental data obtained, it was established that the anticorrosive efficiency depends on the nature of the organic and inorganic component, and the proportions of the systems.

The regularities established during the work expand and complement the existing ideas about the protective effect of two-component organic-inorganic systems. The results will significantly contribute to the creation of effective, environmentally friendly and economically viable steel corrosion inhibitors.

\section{References}

1. E. McCafferty, (20100, Introduction to Corrosion Science, Springer, New York.

2. J. Vosta and J. Eliasek, (1971), Corros. Sci., 11, P. 223

3. N.P. Zhuk. (1976), Course in the Theory of Corrosion and Protection of Metals, Moscow, Metallurgy, $8 \mathrm{p}$.

4. G.G. Ulig., R.U. Revi. (1985) Corrosion and the fight against it. Introduction to corrosion science and technology: Per. From the English / Ed. A.M. Sukhotin. - L .: Chemistry, 1989, Trans. Ed., USA, $456 \mathrm{p}$.

Int. j. biol. chem. (Online)
5. Bregman J.I. (1966), Inhibitors of corrosion, Moscow: Chemistry, $312 \mathrm{p}$.

6. S.M. Reshetnikov. (1986), Inhibitors of acid corrosion of metals, L.: Chemistry, 116 p.

7. Vladimirskaya T.N., Chepelevetskii M.L. (1989), Chemistry and Technology of Condensed Phosphates, p.158-162.

8. Niyazbekova A.B., Akatyev N.V., Sulekeshova G.K., Shakirov T.A. (2013), Chromatographic study of systems cyclotri-, cyclotetra- and cyclohexaphosphate with two and trivalent cations of $\mathrm{p}$ - and d-elements. Technology «Materials of the vi international research and practice conference», Munich, Germany

9. Kuanysheva G.S., Makasheva G.R., Kamalova G., Niyazbekova A.B. (1999), Bull. of KazGU, №1 (13), p.71-73.

10. Corbridje D.E.C. The structural of chemistry of phosphates (1971), Bull. soc. fr. miner. et. cristallog, Vol. 94, No. 1, P. 271-299.

11. Zhdanov U.F. (1979), Chemistry and technology of polyformings, Moscow: Khimiya, $240 \mathrm{p}$.

12. Lazerov A.B., Kubasova L.B., Chudinova N.N. (1982), Receipts and researches and phosphates of alkaline metals, Vol, 18, No. 9, P. 127-131.

13. André Durif. (2005), Solid State Sciences, Vol. 7, Iss. 6, P. 760-766.

14. Pearson R. (1974), Successes of chemistry, Vol. 15, No. 7, P. 1259-1271.

15. Tananayev I.B., Lavrov A.B., Chudinova N.N. (1988), Research of phosphates, Vol. 33, No. 5, P. 2041-2048.

16. Narayana N., María Rao, Gómez-García R., Kornberg A. (2009), An-nual Review of Biochemistry, Vol. 78, P. 605-647.

17. Karapetov M.Kh., Drakin C.I. (1981), General and Inorganic Chemistry, Moscow: Khimiya, 428 p.

18. Genichiro Kura. (1987), Polyhedron, Vol. 6, Iss. 3, P. 531-533. 